\title{
Perioperative Cardiovascular Assessment of Patients Undergoing Noncardiac Surgery
}

\author{
William K. Freeman, MD, and Raymond J. Gibbons, MD
}

\begin{abstract}
Preoperative assessment of the cardiac patient before noncardiac surgery is common in the clinical practice of the medical consultant, anesthesiologist, and surgeon. Currently, most noncardiac surgical procedures are performed for patients of advanced age, and the number of such surgeries is likely to increase with the aging of the population. These same patients have an increased prevalence of cardiovascular disease, especially ischemic heart disease, which is the primary cause of perioperative morbidity and mortality associated with noncardiac surgery. Since 1996, 3 American College of Cardiology/American Heart Association guideline documents have been published, each reflecting the available literature, with recommendations for the preoperative cardiovascular evaluation and treatment of the patient undergoing noncardiac surgery. Our review describes the 2007 American College of Cardiology/American Heart Association guidelines, the most recent revision, focusing on a newly recommended 5-step algorithmic approach to managing this clinical problem, particularly for the patient with known or suspected coronary heart disease. Continued emphasis should be given to preoperative clinical risk stratification, with noninvasive testing reserved for those patients in whom a substantial change in medical management would be anticipated based on results of testing. Pharmacologic therapy holds more promise than coronary revascularization for the reduction of major adverse perioperative cardiac events that might complicate noncardiac surgery.
\end{abstract}

Mayo Clin Proc. 2009;84(1):79-90

ACC = American College of Cardiology; AHA = American Heart Association; DECREASE = Dutch Echocardiographic Cardiac Risk Evaluation Applying Stress Echocardiography; ECG = electrocardiogram; $\mathrm{HR}=$ hazard ratio; $\mathrm{MET}=$ metabolic equivalent; $\mathrm{OR}=$ odds ratio; $\mathrm{PCI}=$ percutaneous coronary intervention; POISE = Perioperative Ischemic Evaluation

$\mathrm{P}$ atients who undergo noncardiac surgery may be at risk for cardiac morbidity and mortality, not only intraoperatively but also during their recovery period. This risk applies particularly to those patients with known cardiac or cerebrovascular disease; however, it may also apply to asymptomatic persons who are older than 50 years and who have the potential to develop atherosclerotic cardiovascular disease. The preoperative assessment of such patients was the subject of the American College of Cardiology/American Heart Association (ACC/AHA) clinical practice guidelines published in 1996. ${ }^{1}$ These guidelines were subsequently updated in $2002^{2}$ and extensively revised in $2007 .^{3}$

As evidence-based clinical cardiologists, we have each had a personal interest in this matter. One of us (W.K.F.) served on the writing committee for the ACC/AHA 2007 Guidelines on Perioperative Cardiovascular Evaluation and Care for Noncardiac Surgery. In addition, we have both been involved with an effort at Mayo Clinic's site in Roch- ester, MN, to improve the quality of perioperative cardiovascular care of patients who undergo noncardiac surgery.

Our goal in writing this focused overview of the ACC/ AHA 2007 guidelines $^{3}$ was to facilitate their more widespread adoption in clinical practice. By far, the most common cardiac issue confronted by the clinician during preoperative evaluation of patients for noncardiac surgery is ischemic heart disease. Thus, this issue is the primary emphasis of this review. Beyond the scope of this review are summaries of all the relevant literature on this topic or of the ACC/AHA recommendations for every aspect of perioperative cardiovascular care. The interested reader is referred to the $\mathrm{ACC}^{4}$ and $\mathrm{AHA}^{5}$ Web sites, where the complete published version of the 2007 guidelines is available.

We have reproduced, with permission, several important tables and a figure from the ACC/AHA 2007 guidelines $^{3}$ because they represent the best-available consensus of the ACC and AHA regarding the critical issues encountered in this common clinical situation. Class recommendations in the ACC/AHA 2007 guidelines $^{3}$ are supplemented by reference to level of evidence, based on available literature, regarding the effect of a specific treatment or procedure (Table 1).

Although the details of many of the figures, tables, and recommendations in the ACC/AHA guidelines have changed between 1996 and 2007 to reflect the increasing scientific evidence relevant to this clinical problem, the overall principles have not changed substantially since the guidelines were first published.

The 3 major parameters that help to determine the risk of cardiac morbidity and mortality for patients who undergo noncardiac surgery and, therefore, help determine the need for additional testing and specific pharmacologic therapy before and during the planned surgery are as follows: (1) the clinical characteristics of the patient, (2) the inherent cardiac risk of the planned surgical procedure, and (3) the patient's functional capacity. It is important to recognize at the outset that these 3 general concepts-patient clinical

From the Division of Cardiovascular Diseases, Mayo Clinic, Rochester, MN.

Address correspondence to William K. Freeman, MD, Division of Cardiovascular Diseases, 200 First St SW, Rochester, MN 55905 (freeman.william @mayo.edu). Individual reprints of this article and a bound reprint of the entire Symposium on Cardiovascular Diseases will be available for purchase from our Web site www.mayoclinicproceedings.com.

() 2009 Mayo Foundation for Medical Education and Research 
TABLE 1. Class Recommendations and Levels of Evidence (LOEs)

\begin{tabular}{|c|c|}
\hline \multicolumn{2}{|l|}{ Class } \\
\hline I & Benefit $\gg \gg$ risk; treatment is recommended and indicated \\
\hline IIa & $\begin{array}{l}\text { Benefit >> risk; reasonable to perform treatment, which is likely } \\
\text { to be effective and beneficial }\end{array}$ \\
\hline $\mathrm{IIb}$ & $\begin{array}{l}\text { Benefit } \geq \text { risk; treatment might be considered, but benefit is } \\
\text { less well established }\end{array}$ \\
\hline III & Risk $\geq$ benefit; treatment not recommended, may be harmful \\
\hline \multicolumn{2}{|l|}{ LOE } \\
\hline A & $\begin{array}{l}\text { Sufficient evidence from multiple population risk studies, } \\
\text { randomized trials, or meta-analyses }\end{array}$ \\
\hline B & $\begin{array}{l}\text { Generally supportive evidence from limited population risk } \\
\text { studies, randomized trials, or meta-analyses }\end{array}$ \\
\hline $\mathrm{C}$ & $\begin{array}{l}\text { Very limited literature support or expert/committee consensus } \\
\text { opinion }\end{array}$ \\
\hline
\end{tabular}

characteristics, risk of surgery, and functional capacityhave withstood the test of time in clinical practice over the past 10 years and remain the critical compoments of defining this problem in any individual patient.

According to the ACC/AHA 2007 guidelines, ${ }^{3}$ the clinical assessment of the patient should identify serious active cardiac conditions, which must be evaluated and managed before surgery to prevent their possible preclusion of surgery in the immediate future. In previous guidelines, these conditions were termed major risk predictors. The clinical assessment should also include a consideration of clinical risk factors, which identify underlying cardiac disease that may also affect the need for additional testing or specific pharmacologic therapy. In previous guidelines, these factors were called intermediate risk predictors. This review provides a detailed discussion of both active cardiac conditions and clinical risk factors.

Both the 1996 guidelines and the 2002 update by the ACC and AHA included a series of algorithms to define clinical decision making in a variety of scenarios. These diagrams were summarized on a series of pocket cards and pocket guidelines that became popular among physicians throughout the United States. However, some clinicians found these diagrams to be too complicated, thereby hindering the more widespread adoption of the guidelines. These previous algorithms also advocated routine stress testing, coronary angiography, and revascularization for certain subgroups of patients; however, results of subsequent studies have put the benefits of such approaches into question.

The 2007 revision of the ACC/AHA guidelines ${ }^{3}$ attempted to address these shortcomings by constructing a single algorithm that delineates 5 key steps in preoperative clinical assessment (Figure). One of the authors (W.K.F.) contributed to the development of this figure. The other author (R.J.G.) has used this figure in his own clinical practice, in the education of cardiac fellows and medical residents, and in formal lectures on this subject. The au- thors consider this algorithm to be a user-friendly, readily applicable, important resource for the practicing clinician who is conducting the cardiovascular assessment of a patient who is undergoing noncardiac surgery.

The current review proceeds through the sequence of 5 steps outlined in the Figure from the ACC/AHA 2007 guidelines. ${ }^{3}$ The most common cardiac issue prompting concern regarding risk in patients undergoing noncardiac surgery is coronary heart disease. Such concern is appropriate, as most cardiovascular morbidity and mortality are related to ischemic heart disease. ${ }^{3}$

\section{STEP 1: IS THERE CLINICAL NEED FOR EMERGENCY NONCARDIAC SURGERY?}

A variety of emergent, life-threatening cardiovascular conditions require emergency surgical intervention. Examples include a ruptured abdominal aortic aneurysm, an acute subdural hematoma with papilledema, and any posttraumatic event with resulting life-threatening conditions. In such patients, noncardiac surgery should proceed as soon as possible, without extensive cardiac assessment. Such preoperative cardiac testing may not only delay potentially life-saving surgery but also yield results that cannot be immediately addressed.

Recommendations for perioperative surveillance and management are appropriate in patients identified as having substantial preexisting cardiac conditions. One example would be a recommendation to continue and closely monitor $\beta$-blocker therapy (including intravenous administration, if necessary) in patients who have known ischemic heart disease and who have been using $\beta$-blockers on a long-term basis. However, further cardiac evaluation and risk-factor management must be deferred to the postoperative period.

\section{STEP 2: ARE THERE ACTIVE CARDIAC CONDITIONS?}

Certain unstable or potentially unstable cardiac conditions dramatically increase the risk of cardiac morbidity and mortality. As previously mentioned, these conditions are referred to as active cardiac conditions in the latest $\mathrm{ACC} /$ AHA guidelines (Table 2). ${ }^{3}$ Such conditions include unstable coronary syndromes, decompensated heart failure, substantial arrhythmias, and severe mitral stenosis or aortic stenosis. Except when emergency noncardiac surgery is warranted, these active conditions preclude proceeding with noncardiac surgery without further evaluation and management of the cardiac problem. Such cardiac conditions are usually readily apparent from the patient's clinical history, physical examination findings, and electrocardiogram (ECG) and chest radiograph results. 


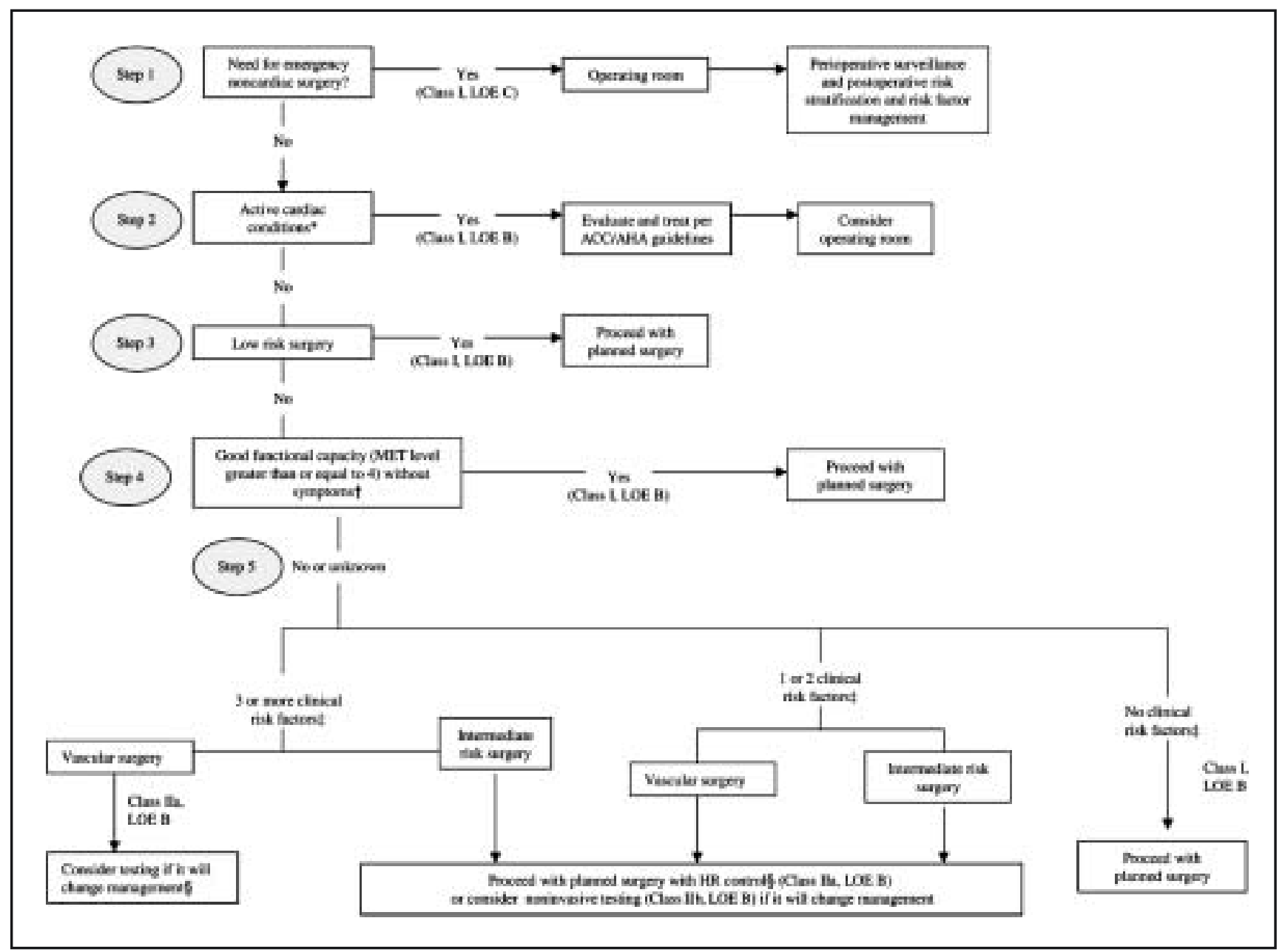

FIGURE. Cardiac evaluation and care algorithm for patients undergoing noncardiac surgery, based on active cardiac conditions, known cardiovascular disease, and cardiac risk factors in patients aged 50 years or older. ACC = American College of Cardiology; AHA = American Heart Association; HR = heart rate; $\mathrm{LOE}$ = level of evidence; $\mathrm{MET}=$ metabolic equivalent.

* See Table 2 for active cardiac conditions and Table 3 for cardiac risk levels of noncardiac surgical procedures.

$\dagger$ See Table 4 for estimated MET levels.

\# See Table 5 for clinical risk factors, which include ischemic heart disease, compensated previous heart failure, cerebrovascular disease, diabetes mellitus, and renal insufficiency.

$\S$ See Table 6 for recommendations for using perioperative $\beta$-blockade with patients in whom this therapy has been shown to reduce cardiac morbidity/mortality.

From J Am Coll Cardiol, ${ }^{3}$ with permission from Elsevier.

In selected patients, echocardiography may be required to confirm the presence of severe valvular disease or to better categorize the severity and type of heart failure. The presence of 1 or more active cardiac conditions, as delineated in Table 2, warrants consultation with a cardiologist. Evaluation and management of these conditions should proceed according to the ACC/AHA 2007 guidelines $^{3}$ before proceeding with any elective noncardiac surgery. Preoperative management is often dependent on individual patient circumstances. Neither operative coronary intervention nor percutaneous coronary intervention $(\mathrm{PCI})$ is usually necessary to lower the risk of noncardiac surgery, unless such intervention is indicated for the overall benefit of the patient irrespective of the preoperative context.

A review of the evaluation and therapy associated with each of the active conditions listed in Table 2 is beyond the scope of this article.

\section{STEP 3: DOES THE PLANNED SURGERY HAVE A LOW CARDIAC RISK?}

As already mentioned, 1 of the 3 key issues in perioperative assessment of patients undergoing noncardiac surgery is the inherent risk of the surgical procedure. Surgi- 
TABLE 2. Active Cardiac Conditions for Which the Patient Should Undergo Evaluation and Treatment Before Noncardiac Surgery ${ }^{a}$

\begin{tabular}{|c|c|}
\hline Condition & Examples \\
\hline $\begin{array}{l}\text { Unstable coronary } \\
\text { syndromes }\end{array}$ & $\begin{array}{l}\text { Unstable or severe angina (CCS class III } \\
\text { or IV) } \\
\text { Recent myocardial infarction (7-30 d) }\end{array}$ \\
\hline $\begin{array}{l}\text { Decompensated heart } \\
\text { failure (NYHA functional } \\
\text { class IV; worsening or } \\
\text { new-onset heart failure) }\end{array}$ & $\begin{array}{l}\text { High-grade atrioventricular block } \\
\text { Mobitz II atrioventricular block } \\
\text { Third-degree atrioventricular block } \\
\text { Symptomatic ventricular arrhythmias }\end{array}$ \\
\hline Significant arrhythmias & $\begin{array}{l}\text { Supraventricular arrhythmias } \\
\text { (including atrial fibrillation) with } \\
\text { uncontrolled ventricular rate } \\
\text { (heart rate }>100 \text { beats/min at rest) } \\
\text { Symptomatic bradycardia } \\
\text { Newly recognized ventricular tachycardia }\end{array}$ \\
\hline Severe valvular disease & $\begin{array}{l}\text { Severe aortic stenosis (mean pressure } \\
\text { gradient }>40 \mathrm{~mm} \mathrm{Hg} \text {, aortic valve area } \\
<1.0 \mathrm{~cm}^{2} \text {, or symptomatic) } \\
\text { Symptomatic mitral stenosis (progressive } \\
\text { dyspnea on exertion, exertional } \\
\text { presyncope, or heart failure) }\end{array}$ \\
\hline
\end{tabular}

${ }^{\mathrm{a}} \mathrm{CCS}=$ Canadian Cardiovascular Society; NYHA = New York Heart Association

Adapted from J Am Coll Cardiol, ${ }^{3}$ with permission from Elsevier.

cal procedures that have a reported cardiac risk of less than $1 \%$ for cardiac death and nonfatal myocardial infarction are considered to be low risk in the ACC/AHA 2007 guidelines. ${ }^{3}$ Multiple common surgical procedures in clinical practice fall within this category, including most endoscopic, ophthalmologic, dermatologic, and breast surgical procedures (Table 3). Other minimally invasive surgical procedures that are performed on an ambulatory basis without overnight observation also fall within the low-risk category.

Because the risk of cardiac death and nonfatal myocardial infarction with these procedures is so low, patients being considered for surgery do not generally require further

TABLE 3. Cardiac Risk Stratification for Patients Undergoing Noncardiac Surgery ${ }^{a}$

\begin{tabular}{cl}
\hline \multicolumn{1}{c}{ Risk stratification } & \multicolumn{1}{c}{ Procedure examples } \\
\hline Vascular (reported & Aortic and other major vascular surgery \\
cardiac risk $>5 \%)$ & Peripheral vascular surgery \\
Intermediate risk (reported & Intraperitoneal and intrathoracic surgery \\
cardiac risk 1\%-5\%) & Carotid endarterectomy \\
& Head and neck surgery \\
& Orthopedic surgery \\
& Prostate surgery \\
Low risk (reported & Endoscopic procedures \\
cardiac risk $<1 \%)$ & Superficial procedures \\
& Cataract surgery \\
& Breast surgery \\
& Ambulatory surgery \\
\hline
\end{tabular}

${ }^{a}$ Combined incidence of death and nonfatal myocardial infarction. Adapted from J Am Coll Cardiol, ${ }^{3}$ with permission from Elsevier. preoperative cardiac testing or new pharmacologic therapy. Patients who are currently using $\beta$-blockers should continue using them through the perioperative period.

Unfortunately, patients who are at low cardiac risk with surgery are sometimes referred inappropriately for cardiac assessment, including stress imaging studies. In some cases, this assessment is conducted in response to patient or physician anxiety about the patient's advanced age or comorbidities. A recent Mayo Clinic study of the use of stress myocardial perfusion imaging and stress echocardiography identified low-risk surgery patients as among the leading inappropriate referrals for these tests. ${ }^{6}$ Before the publication of the updated ACC/AHA guidelines in 2007, ${ }^{3}$ such patients constituted $17 \%$ of all indications for inappropriate stress myocardial perfusion imaging and stress echocardiography studies performed at Mayo Clinic's site in Rochester, MN. ${ }^{6}$ Other studies have reported similar findings. ${ }^{7}$

Furthermore, reports of the ACC-Duke University Medical Center Think Tank on Quality in Cardiovascular Imaging $^{8}$ suggest that many stress imaging laboratories have observed similarly high rates of inappropriate referrals for cardiac tests among patients who are at low cardiac risk with surgery. Thus, it is clear that additional perioperative cardiac assessment in such patients is unnecessary, and the results of such assessment could potentially prompt additional inappropriate invasive evaluation and intervention. The apparently widespread practice of inappropriate referrals is strongly discouraged by the current ACC/AHA guidelines. ${ }^{3}$

One question that frequently arises during determination of the cardiac risk of planned surgical procedures is how to assign a surgical risk level to a planned laparoscopic procedure. An increasing number of abdominal and thoracic procedures are now initially attempted with a laparoscopic approach. Some clinicians would argue that the need for follow-up open surgery, if warranted by laparoscopic findings or technical limitations of the laparoscopic approach, would require classification of the planned laparoscopic procedure as intermediate-risk surgery. Application of the ACC/AHA guidelines ${ }^{3}$ to individual patients in these situations must be a matter of clinical judgment, preferably guided by some estimation on the part of the surgeon regarding the likelihood of the further need for open surgery.

If the planned laparoscopic procedure is unlikely to require further, extensive surgical intervention, that patient can be reasonably assigned to the low-risk surgery category, obviating the need for further preoperative cardiac assessment. Because of the absence of substantial evidence-based literature on cardiac outcomes of specific laparoscopic procedures, the current ACC/AHA guidelines $^{3}$ do not provide definitive recommendations regarding laparoscopic surgical risk stratification. 


\section{STEP 4: DOES THE PATIENT HAVE GOOD FUNCTIONAL CAPACITY WITHOUT SYMPTOMS?}

Many studies of cardiac risk for patients undergoing noncardiac surgery have found that the patient's functional status is a reliable predictor of both perioperative and longterm cardiac risk. A good functional capacity is generally defined on the basis of metabolic equivalent (MET) levels. For reference purposes, activities of daily living, such as eating, dressing, toilet use, and showering, typically require 1 to 2 METs, whereas such strenuous sports as swimming, singles tennis, and skiing require more than 10 METs.

In some cases, determination of whether the patient has a good functional capacity will be obvious on the basis of clinical history. A patient who never leaves a single-level house because of cardiac symptoms has a poor functional capacity. By contrast, a patient who regularly plays singles tennis has excellent functional capacity.

In other cases, a more detailed clinical history will be required to determine functional capacity. Simple questions can provide a means of making a reasonable estimate of the patient's functional capacity. Although a review of clinical history may not be as objective as results from exercise treadmill testing, such clinical assessment can be valuable in preoperative risk evaluation. One clinical scale used to determine functional capacity is the Duke Activity Status Index (Table 4), which was proved to be remarkably effective in the preoperative assessment of patients in the health care system of the US Department of Veterans Affairs. ${ }^{9}$

We prefer the use of 2 simple questions to determine a patient's functional capacity: (1) Can you walk 4 blocks without stopping because of limiting symptoms? (2) Can you climb 2 flights of stairs without stopping because of limiting symptoms? An affirmative answer to either of these questions confirms an adequate functional capacity in the patient. Such activities support the patient's tolerance to exertion at a level of 4 to $5 \mathrm{METs}$, which is typically equivalent to the physiologic stress of most noncardiac surgical procedures requiring general anesthesia. By contrast, unequivocally negative responses to both questions are usually sufficient to confirm a poor functional capacity.

Patients with good functional capacity can proceed with their planned surgery without further perioperative assessment or specific pharmacologic therapy. As previously noted, such further assessment is of no incremental value and should be strongly discouraged.

\section{STEP 5: DOES THE PATIENT HAVE CLINICAL RISK FACTORS?}

The term clinical risk factors needs to be precisely defined in order to avoid potential confusion in clinical practice. The
TABLE 4. Estimated Energy Requirements for Various Activities, Based on Duke Activity Status Index

\begin{tabular}{cc}
\hline 1 MET & Can you... \\
& take care of yourself? \\
& eat, dress, or use the toilet? \\
& walk indoors around the house? \\
& walk 1 or 2 blocks on level ground at 2-3 mph \\
& $(3.2-4.8$ kph)? \\
& Can you... \\
& do light work around the house, such as dusting or \\
& washing dishes? \\
& Can you... \\
& climb a flight of stairs or walk up a hill? \\
& walk on level ground at 4 mph (6.4 kph)? \\
& run a short distance? \\
& do heavy work around the house, such as scrubbing \\
& floors or lifting or moving heavy furniture? \\
& participate in moderate recreational activities, such \\
& as golf, bowling, dancing, doubles tennis, or \\
& throwing a baseball or football? \\
& Can you... \\
& participate in strenuous sports, such as swimming, \\
& singles tennis, football, basketball, or skiing?
\end{tabular}

${ }^{\mathrm{a}} \mathrm{MET}=$ metabolic equivalent.

Adapted from J Am Coll Cardiol, ${ }^{3}$ with permission from Elsevier.

clinical risk factors referred to in this article are not the traditional risk factors for atherosclerosis that were originally defined in the Framingham Heart Study (ie, hypertension, hyperlipidemia, tobacco use, diabetes mellitus, family history of premature atherosclerosis). Rather, the clinical risk factors in this article, based on the ACC/AHA 2007 guidelines, ${ }^{3}$ are conditions that have been associated in a variety of studies with an increased risk of cardiac events at the time of noncardiac surgery.

The preoperative clinical cardiac risk factors used in the ACC/AHA 2007 guidelines ${ }^{3}$ are derived from the Revised Cardiac Risk Index, described by Lee et al. ${ }^{10}$ These investigators retrospectively derived predictors of major adverse perioperative cardiac events (ie, nonfatal myocardial infarction, pulmonary edema, complete heart block, cardiac arrest, cardiac death) in a cohort of 2893 patients. They then prospectively validated this index in another cohort of 1422 patients. The following 6 independent predictors were identified in the Revised Cardiac Risk Index ${ }^{10}$ : stable ischemic heart disease, compensated heart failure, cerebrovascular disease, diabetes mellitus requiring preoperative insulin therapy, preoperative creatinine level greater than $2 \mathrm{mg} / \mathrm{dL}$ (to convert to $\mu \mathrm{mol} / \mathrm{L}$, multiply by 88.4 ), and high-risk noncardiac surgery.

The ACC/AHA 2007 guidelines $^{3}$ use the term cardiac risk factors for the first 5 clinical components of the Revised Cardiac Risk Index. ${ }^{10}$ These clinical components, along with specific criteria for each, are summarized in Table 5. In contrast to the Revised Cardiac Risk Index, the ACC/AHA guidelines allow inclusion of diabetes mellitus 
TABLE 5. Clinical Risk Factors That Predict Risk of Cardiac Death and Nonfatal Myocardial Infarction at Time of Noncardiac Surgery ${ }^{a}$

\begin{tabular}{ll}
\hline \multicolumn{1}{c}{ Condition } & \multicolumn{1}{c}{ Examples } \\
\hline History of ischemic heart & Previous myocardial infarction \\
disease & Previous positive result on stress test \\
& Use of nitroglycerin \\
& Typical angina \\
& ECG Q waves \\
& Previous PCI or CABG \\
History of compensated & History of heart failure \\
previous congestive & Previous pulmonary edema \\
heart failure & Third heart sound \\
& Bilateral rales \\
& Evidence of heart failure on chest \\
& radiograph \\
History of cerebrovascular & Previous TIA \\
disease & Previous stroke \\
Diabetes mellitus & With or without preoperative insulin \\
& therapy \\
Renal insufficiency & Creatinine level $>2 \mathrm{mg} / \mathrm{dL}^{\mathrm{b}}$ \\
\hline
\end{tabular}

${ }^{\mathrm{a}} \mathrm{CABG}=$ coronary artery bypass grafting; $\mathrm{ECG}=$ electrocardiographic; $\mathrm{PCI}=$ percutaneous coronary intervention; TIA $=$ transient ischemic attack.

${ }^{\mathrm{b}}$ SI conversion factor: To convert creatinine value to $\mu \mathrm{mol} / \mathrm{L}$, multiply by 88.4

Data from Circulation. ${ }^{10}$

as a clinical cardiac risk factor without the requirement for insulin therapy.

\section{Risk Factors Absent}

If a patient has none of these clinical risk factors, he or she should proceed with the planned noncardiac surgery without further preoperative cardiac assessment. In such cases, the anticipated risk of a major adverse perioperative cardiac event complicating any noncardiac surgical procedure would be approximately $0.5 \% .^{10}$ Thus, the ACC/AHA 2007 guidelines $^{3}$ recommend that a patient with a poor functional capacity but no clinical risk factors proceed directly to vascular surgery without further testing. This recommendation represents a change from the previous versions of the guidelines, which favored preoperative cardiac stress testing in all patients with poor functional capacity who were scheduled to undergo high-risk surgery.

\section{Risk Factors Present}

If the patient has a clinical risk factor, the clinical decisionmaking process is more complex. Until this point in the algorithm (Figure), an affirmative answer to any of the questions leads to either proceeding with the planned surgery (ie, emergency surgery, low-risk surgery, surgery in cases of good functional capacity) or delaying the surgery pending evaluation and management of the active cardiac condition. This point marks the first time in the decisionmaking process at which more extensive perioperative cardiac assessment may be warranted. Patients who are candi- dates for vascular surgery and have 3 or more clinical risk factors comprise a separate high-risk group and are addressed later in this article.

For any patient who is undergoing intermediate-risk surgery and who has at least 1 clinical risk factor or any patient who is undergoing vascular surgery and who has 1 or 2 clinical risk factors, the decision facing the clinician is either to proceed with the planned surgery and use pharmacologic control of heart rate to avoid excessive myocardial oxygen demand or to consider further noninvasive cardiac testing if the results could change subsequent management (Figure). Planned surgery with heart rate control carries a class IIa level of recommendation (ie, it is reasonable to perform and supported by randomized trial results). ${ }^{11} \mathrm{By}$ comparison, further noninvasive testing carries a lower, class IIb recommendation (ie, it may be considered).

\section{$\beta$-BLOCKER THERAPY}

In an attempt to decrease perioperative cardiac morbidity and mortality during noncardiac surgery, a number of pharmacologic therapeutic options have been explored, including use of $\beta$-blockers, statins, $\alpha 2$ agonists, and calcium channel blockers. Clearly, the most extensively studied of these options is $\beta$-blocker therapy. Although previous ACC/AHA guidelines noted the limited published evidence about $\beta$-blockers in an attempt to inspire more studies, there remain few randomized controlled trials of these medications.

Most earlier perioperative trials of $\beta$-blocker therapy involved relatively small numbers of heterogeneous patients who were undergoing a wide variety of surgical procedures. A broad spectrum of $\beta$-blocker regimens was used in these trials, often without protocols for titration to drug effect before noncardiac surgery. This high degree of heterogeneity in patient populations and study design has important implications when multiple small studies cumulatively undergo meta-analysis. These meta-analyses have yielded a variance of opinion regarding the efficacy of $\beta$ blocker therapy in patients undergoing noncardiac surgery. ${ }^{12-14}$ Therefore, the conclusions of such meta-analyses must be interpreted with caution.

In addition to the small number of randomized controlled trials, other major limitations of the available literature on $\beta$-blockers include limited data comparing different $\beta$-blocker agents, as well as a lack of guidance regarding such practical issues as timing, location, and route of administration of perioperative $\beta$-blocker therapy.

A general consensus maintains that $\beta$-blockers should be continued in patients undergoing surgery who are receiving $\beta$-blockers to treat angina, symptomatic arrhythmias, hypertension, or other ACC/AHA class I guideline 
TABLE 6. Recommendations for Perioperative $\boldsymbol{\beta}$-Blocker Therapy Based on Published Randomized Clinical Trials ${ }^{a}$

\begin{tabular}{|c|c|c|c|c|}
\hline Surgery $^{b}$ & $\begin{array}{l}\text { No clinical } \\
\text { risk factors }\end{array}$ & $\begin{array}{l}1 \text { or more clinical } \\
\text { risk factors }\end{array}$ & $\begin{array}{c}\text { CHD or } \\
\text { high cardiac risk }\end{array}$ & $\begin{array}{l}\text { Patients currently } \\
\text { taking } \beta \text {-blockers }\end{array}$ \\
\hline Vascular & Class IIb, LOE B & Class IIa, LOE B & $\begin{array}{l}\text { Patients found to have } \\
\text { myocardial ischemia on } \\
\text { preoperative testing: } \\
\text { class I, LOE } \mathrm{B}^{\mathrm{d}} \\
\text { Patients without ischemia } \\
\text { or no previous test: } \\
\text { class IIa, LOE B }\end{array}$ & Class I, LOE B \\
\hline Intermediate-risk & $\ldots$ & Class IIb, LOE C & Class IIa, LOE B & Class I, LOE C \\
\hline Low-risk & $\ldots$ & $\ldots$ & $\ldots$ & Class I, LOE C \\
\hline \multicolumn{5}{|c|}{$\begin{array}{l}{ }^{a} \text { CHD = coronary heart disease; LOE = level of evidence. Ellipses indicate data were insufficient to determine a class } \\
\text { of recommendation or LOE. } \\
{ }^{\text {b }} \text { See Table } 3 \text { for surgical procedural risks. } \\
{ }^{c} \text { See Table } 5 \text { for clinical risk factors. } \\
\text { d Applies to patients found to have coronary ischemia on preoperative testing. } \\
\text { Adapted from } J \text { Am Coll Cardiol, }{ }^{3} \text { with permission from Elsevier. }\end{array}$} \\
\hline
\end{tabular}

indications. Clinical practice at Mayo Clinic has long favored this approach, with recommendations that $\beta$-blocker therapy be administered on the day of surgery and continued with the least possible interruption throughout the perioperative period. Nevertheless, physicians performing cardiac assessments before noncardiac surgery and surgeons performing these procedures need to recognize that many patients do not consistently receive their $\beta$-blockers despite the best of intentions. Such patients prompted a quality improvement effort at Mayo Clinic's site in Rochester, MN, in recognition of the fact that our system of $\beta$ blocker delivery was far less than ideal (D. M. Manning, $\mathrm{MD}$, personal communication).

Interruption of $\beta$-blocker therapy in such patients may lead to recurrent angina, arrhythmias, rebound hypertension, and rapid atrial fibrillation in the perioperative period, precisely when the patient is most vulnerable to additional physiologic stress. We encourage all readers to review the current system of care within their own local hospitals to make certain that the use of $\beta$-blockers is not interrupted in such patients.

Similarly, unless there is a specific perioperative contraindication, other previously prescribed cardiovascular medications should be continued with the least possible interruption throughout the patient's noncardiac surgery perioperative period. If permitted by the surgeon, aspirin should be continued in patients who have had previous surgical or percutaneous coronary revascularization, recognizing the propensity for increased platelet aggregation during noncardiac surgery.

Medications previously used to control conditions such as hypertension, heart failure, and arrhythmias should likewise be continued because exacerbation of these problems could complicate the patient's perioperative course. Con- tinuation of statin therapy carries a class I recommendation $^{3}$ because a reduction in adverse perioperative cardiac events has been observed with statin therapy in a number of retrospective studies, ${ }^{15}$ a case-control study, ${ }^{16}$ and a small randomized controlled trial. ${ }^{17}$

Evidence supporting the efficacy of $\beta$-blockers for other types of patient situations is variable. The strongest evidence for $\beta$-blocker efficacy exists for patients undergoing vascular surgery who are at high cardiac risk because they are found to have myocardial ischemia on preoperative testing. The weakest evidence for $\beta$-blocker efficacy exists for patients undergoing intermediate-risk surgery who have at least 1 clinical risk factor and for patients undergoing vascular surgery who have no clinical risk factors. Table 6 summarizes class recommendations of the ACC/AHA $2007^{3}$ guidelines committee for perioperative $\beta$-blocker therapy, with recommendations listed according to clinical and surgical procedural risk. Interested readers are referred to the guidelines for a more complete discussion of the evidence.

Perioperative $\beta$-blocker therapy must be tailored to the individual patient. Administration of a $\beta$-blocker, especially when the initial dose is low, cannot be assumed to provide therapeutic $\beta$-blockade (such as a resting heart rate of $<65$ beats/min). Many studies show that the efficacy of $\beta$ blockers and the serum levels of patients using $\beta$-blockers vary substantially depending on the individual patient. A considerable portion of this variability is caused by the variable metabolic effects produced by $\beta$-blockers during their first pass through the hepatic venous circulation after absorption in the bowel.

Ideally, the adequacy of $\beta$-blockade would be assessed by the body's chronotropic response to exercise or catecholamine stimulation. However, such assessment is often 
impractical and has not been proven to be cost-effective. The general practical approach to $\beta$-blockade assessment involves measurement of the resting heart rate. Several studies have shown that control of the resting heart rate to less than 65 beats/min is associated with lower cardiac risk during noncardiac surgery. In patients currently taking $\beta$ blockers for the indications previously mentioned, the dosage can be adjusted if necessary to achieve a heart rate of less than 65 beats/min. In patients who are not currently taking $\beta$-blockers and who are judged to merit treatment with $\beta$-blockers to reduce their cardiac risk for noncardiac surgery, the titration of the dosage can pose a logistical problem, depending on the time interval before surgery.

In our experience, as is likely common in other tertiary care institutions, many patients presenting for preoperative medical evaluation are scheduled to undergo surgery shortly thereafter. In such situations, the clinician is forced to make an educated guess regarding the proper $\beta$-blocker dose for a particular patient and then to initiate therapy as soon as possible, relying on the anesthesiologist during the surgery to administer intravenous $\beta$-blockade as required to control the heart rate.

We sense that the starting doses of $\beta$-blockers in general clinical practice have gradually declined in recent years in an effort to avoid the occasional case of symptomatic bradycardia. Low initial doses are permissible in long-term management because the doses can be gradually titrated upward as required. However, the luxury of time may not be available for some patients before noncardiac surgery. Thus, we suggest that clinicians be somewhat more aggressive in their choice of the initial dose of a $\beta$-blocker to make certain that the patient's heart rate is adequately controlled during both the operative and perioperative period.

Patients who begin taking $\beta$-blockers in anticipation of noncardiac surgery must be carefully followed up throughout the perioperative period to make certain that their dosing is adequate and not excessive. The gradual increase in serum levels that may occur over time, particularly in the presence of a long-acting $\beta$-blocker or other drugs or conditions that may interfere with the metabolism of the $\beta$-blocker, can lead to symptomatic hypotension and bradycardia.

The potential hazards of $\beta$-blocker therapy are emphasized by the recently published results of the Perioperative Ischemic Evaluation (POISE) trial. ${ }^{18}$ This multicenter, randomized, placebo-controlled trial involved 8351 patients who underwent surgery that was deemed to be intermediate or high risk by their physicians. Patients who were previously taking $\beta$-blockers or whose physicians planned to start such therapy were excluded. Patients were randomized to receive either placebo or a nontitrated, fixed dose of $100 \mathrm{mg}$ of metoprolol, which was administered 2 to 4 hours before surgery and again within 6 hours after surgery. A maintenance dosage of $200 \mathrm{mg} / \mathrm{d}$ of the drug was administered postoperatively for 30 days. Patients were continued on this dosage as long as their heart rate remained at least 50 beats/min and their systolic blood pressure exceeded $100 \mathrm{~mm} \mathrm{Hg}$.

In the POISE trial, ${ }^{18}$ the primary composite end points of cardiovascular death, cardiac arrest, and nonfatal myocardial infarction were significantly reduced with metoprolol therapy vs placebo (5.8\% vs $6.9 \%$, respectively; hazard ratio [HR], $0.84 ; P=.04)$, mostly as a result of reduction in the rate of nonfatal myocardial infarction $(3.6 \%$ vs $5.1 \%$, respectively; HR, 0.70; $P<.001)$. Total 30-day mortality rate, however, was greater in the metoprolol-treated patients than in the placebo group $(3.1 \%$ vs $2.3 \%$, respectively; HR, $1.33 ; P=.03)$, as was the incidence of clinically important hypotension ( $15 \%$ vs $9.7 \%$; HR, $1.55 ; P<.001)$, severe bradycardia $(6.6 \%$ vs $2.4 \%$; HR, $2.74 ; P<.001)$, and stroke ( $1.0 \%$ vs $0.5 \%$; HR, $2.17 ; P=.005)$.

Stroke (odds ratio [OR], 18.97), hypotension (OR, 4.97), and bradycardia (OR, 2.13) were all predictors of increased mortality and accounted for over half of the populationattributable risk of death in the POISE trial. ${ }^{19}$ Hypotension was a significant predictor of stroke, but identified risk factors could explain only half of the strokes. As Fleisher and Poldermans ${ }^{19}$ point out in an editorial accompanying the POISE trial, the $1.0 \%$ rate of stroke observed in this trial was far greater than the $0.4 \%$ rate observed for the 3994 patients in the Dutch Echocardiographic Cardiac Risk Evaluation Applying Stress Echocardiography (DECREASE) series of trials, in which $\beta$-blocker doses were titrated.

Although $\beta$-blocker therapy is an effective means of reducing the risk of major adverse cardiac events (primarily those related to ischemic heart disease) during noncardiac surgery, the results of the POISE study ${ }^{18}$ underscore the need for careful preoperative titration of a $\beta$-blocker in the individual patient, as well as the importance of postoperative clinical surveillance of patients using this therapy. As shown by the POISE study, ${ }^{18}$ high-dose $\beta$-blocker therapy initiated immediately before noncardiac surgery is associated with a significant incidence of adverse effects that could be anticipated. Specifically, clinically important hypotension and severe bradycardia occurred, and these effects were in turn associated with increased 30-day perioperative mortality. Unfortunately, avoiding these problems with implementation of $\beta$-blocker therapy titrated to optimal effect before noncardiac surgery remains a challenge in routine clinical practice.

\section{CORONARY REVASCULARIZATION}

As previously indicated, noninvasive testing may be considered if the test results have potential to change patient 
management. Because appropriately administered $\beta$ blocker therapy is reasonable (ie, class IIa recommendation) for any patient with at least 1 clinical risk factor at this step in the cardiac evaluation and care algorithm (Figure), the rationale for noninvasive testing is based on the possible discovery of a need for coronary revascularization. Vascular surgical procedures, particularly aortoiliac and peripheral vascular surgery (other than carotid endarterectomy), carry a high risk ( $>5 \%)$ of adverse perioperative cardiac events. Vascular disease is strongly associated with concomitant coronary heart disease as a result of the presence of the same multiple contributing risk factors. In other words, symptoms of myocardial ischemia may not be clinically evident because of functional limitations related to peripheral arterial occlusive disease.

Moreover, major open vascular surgery is associated with substantial shifts in intravascular volume, causing labile changes in both systemic and intracardiac pressures. Patients undergoing such surgery warrant conscientious preoperative evaluation and are likely to be the patients who benefit the most from selective preoperative risk stratification with stress testing, provided that the test results will affect management (Figure).

Stress testing is not recommended as an incremental means of cardiac risk reduction in patients who are at intermediate clinical risk (ie, 1-2 risk factors), even in those who are undergoing major vascular surgery, provided that they are receiving $\beta$-blocker therapy with complete heart rate control. In a study of 770 such patients who were randomized to stress testing vs no stress testing, Poldermans at $\mathrm{al}^{11}$ found no significant difference in rate of perioperative cardiac death or nonfatal myocardial infarction (2.3\% vs $1.8 \%$, respectively; $P=.62)$. This lack of difference was particularly pronounced when heart rate was maintained at less than 65 beats $/ \mathrm{min}$.

If a noninvasive stress test, with or without imaging, yields severely abnormal results, the likelihood of extensive and prognostically important coronary heart disease increases. Patients with such findings are generally referred for coronary angiography. The indications for subsequent coronary bypass surgery or PCI in such patients are the same as in patients with chronic angina. Coronary revascularization is useful in patients with substantial left main coronary heart disease and patients with 3-vessel coronary heart disease (particularly if the ejection fraction is abnormal), as well as in selected patients with 2-vessel coronary heart disease and stenosis of the proximal left anterior descending coronary artery. Such revascularization could have a long-term survival benefit for patients, but it requires a short-term delay in their noncardiac surgery.

After coronary artery bypass grafting, a delay of 4 to 8 weeks is typical before a patient can proceed with noncar- diac surgery. After PCI with bare metal stenting, 30 days of thienopyridine therapy with aspirin is recommended. To minimize the risk of surgical bleeding associated with such therapy, a minimum of 5 days would be required after stopping thienopyridine use before proceeding with noncardiac surgery. After PCI with a drug-eluting stent, dual antiplatelet therapy with aspirin and a thienopyridine is recommended for 1 year. Hence, if PCI is clinically indicated before a noncardiac surgical procedure that needs to be pursued in a timely manner (ie, after 35 days), bare metal stent implantation rather than drug-eluting stent implantation is recommended, unless the bleeding risk of the surgical procedure with use of dual antiplatelet therapy is low enough to permit this therapy to be continued without interruption.

Premature interruption in use of a thienopyridine (eg, clopidogrel or, rarely, ticlopidine) after PCI exposes the patient to a substantial risk of acute stent thrombosis, which carries a high mortality rate. This risk is particularly pronounced for patients who have a history of stent thrombosis and for patients who have high-risk coronary stent locations (eg, left main coronary artery), graft conduit stents, multivessel coronary stents, or a stented single remaining coronary artery. Aspirin should be continued throughout the perioperative period in the patient who has had any type of previous coronary revascularization, unless there is a major contraindication. Before pursuing stress testing, the clinician should carefully weigh the potential downstream consequences of positive study findings, ie, the subsequent implications for revascularization (particularly PCI), if substantial coronary heart disease is found on angiography.

The basic premise of this approach (ie, the noninvasive identification of patients with severe ischemia who warrant revascularization to reduce their risk of noncardiac surgery) has been challenged and somewhat weakened by the results of recent randomized controlled trials. The first large randomized controlled trial of coronary revascularization before vascular surgery was the Coronary Artery Revascularization Prophylaxis (CARP) trial, ${ }^{20}$ in which 258 patients were randomized to revascularization and 252 patients to no revascularization. All the patients had multiple clinical risk criteria, and three-quarters of the patients had moderate to large ischemic burden results on stress imaging. In addition, all the patients had stenosis with 70\% narrowing of at least 1 coronary artery that was suitable for revascularization, but only one-third of the patients had 3vessel coronary heart disease.

Somewhat surprisingly, results of the CARP triall ${ }^{20}$ showed no significant difference between the revascularization group and the no revascularization group in terms of the primary end points of death or myocardial infarction at either 30-day or 2.7-year follow-up. Approximately $85 \%$ 
of patients in both the revascularization and no revascularization groups were receiving $\beta$-blocker therapy during vascular surgery, a factor that may have affected the potential incremental benefit of coronary revascularization. ${ }^{20}$

The investigators of the DECREASE-V pilot study ${ }^{21}$ identified 101 patients who were scheduled for major vascular surgery, who had 3 or more clinical risk factors, and who had extensive ischemia on stress imaging studies. These patients were randomized to receive either revascularization or optimal $\beta$-blocker therapy with a target heart rate control of less than 65 beats/min. In this high-risk surgery population, no difference was observed in the rate of 30-day all-cause death or nonfatal myocardial infarction between the revascularization and $\beta$-blocker groups $(43 \%$ vs $33 \%$, respectively; OR, $1.4 ; 95 \%$ confidence interval, $0.7-2.8 ; P=.30)$. Neither were significant differences observed between the groups in 1-year outcomes. Although the DECREASE-V pilot study ${ }^{21}$ was inadequately powered to definitively establish the value of preoperative revascularization, the results were consistent with previous observational studies that suggested a lack of benefit for this procedure.

A rationale for noninvasive testing still exists, as reflected in the class IIb recommendation in the ACC/AHA 2007 guidelines. ${ }^{3}$ However, stress testing should no longer be regarded as a routine step in preoperative evaluation of even high-risk patients, unless test results have the potential to substantially affect patient management. Such results may prompt modification of pharmacologic therapy, perioperative surveillance, and postoperative follow-up; however, they should not automatically trigger momentum toward preoperative coronary revascularization, which remains an unproven means of reducing cardiac risk during noncardiac surgery. We would like to again emphasize that patients with active cardiac conditions, such as unstable coronary syndromes or recent myocardial infarction, must be managed early in the 5-step algorithm process (Figure) per AHA/ACC guidelines. ${ }^{22}$

One practical question that frequently arises if the noninvasive testing option is pursued is which preoperative stress test to use. In patients with normal ECG results who are able to exercise, the preferred stress test is exercise ECG testing. This testing not only identifies patients with severe ischemia but also objectively quantifies functional capacity if this capacity cannot be assessed by clinical history (as described in the section on step 3). In patients with abnormal resting ECG results that preclude interpretation of the exercise ECG test, stress cardiac imaging is indicated. In patients who are unable to perform adequate exercise, pharmacologic stress imaging will be necessary. All of these imaging modalities have published criteria to help clinicians define severely abnormal test results.
If the clinician thinks that revascularization is unequivocally necessary, he or she should carefully consider the timing and need for noncardiac surgery in selecting the type of revascularization to be used. The recognized importance of dual antiplatelet therapy after stent placement must be incorporated into this decision. Because the interruption of dual antiplatelet therapy after implantation of drug-eluting stents increases the risk of stent thrombosis during the first year after stent placement (particularly during the first 3 months after sirolimus-eluting stent placement and the first 6 months after paclitaxel-eluting stent placement), drug-eluting stents are the less desirable option before noncardiac surgery. ${ }^{23}$ By contrast, bare metal stents permit interruption of dual antiplatelet therapy after only 1 month without undue risk. However, bare metal stents carry an increased risk of long-term restenosis. Coronary bypass surgery is associated with greater morbidity and a more prolonged recovery time than stent placement. Some elderly patients never recover sufficiently from bypass surgery to proceed with noncardiac surgery.

Not uncommonly, patients may present for noncardiac surgery with a history of 1 or more coronary revascularization procedures. Multiple studies have found that patients who have undergone coronary artery bypass grafting and who are asymptomatic or clinically stable with only mild symptoms can be managed with the same approach as previously outlined. Obviously, these patients have at least 1 clinical risk factor because they have evidence of previous coronary heart disease. If patients do not have good functional capacity and are undergoing intermediate-risk or high-risk surgery, they merit treatment with $\beta$-blockers. Although noninvasive testing may identify mild ischemia in patients, the likelihood of detecting severe ischemia warranting additional revascularization is less in those patients than in patients who have not undergone cardiac evaluation.

In patients who are clinically stable after coronary revascularization and who are receiving appropriate pharmacologic therapy, noninvasive testing is unlikely to substantially change the approach to their management. Such patients should be receiving long-term antiplatelet therapy, usually with aspirin alone. Although many surgeons prefer to routinely stop aspirin to minimize bleeding risk, we advise against this practice in patients with previous coronary revascularization. In many of these patients, the cardiac risk associated with interruption of aspirin use exceeds the risk of major bleeding.

In patients who have undergone cutaneous coronary intervention with stenting, considerations regarding interruption of dual antiplatelet therapy are important. Patients who have had bare metal stenting more than 30 days previously and those who have had drug-eluting stenting more 
than 1 year previously can generally have their thienopyridine safely discontinued. However, such patients should also be taking a single antiplatelet agent, usually aspirin, on a long-term basis. The risk of interrupting this therapy for noncardiac surgery must be weighed against the increased bleeding associated with aspirin. We generally advise our surgical colleagues to have their patients continue using aspirin if at all possible and to restart aspirin as soon as possible if its use is interrupted.

\section{POSTOPERATIVE SURVEILLANCE FOR MYOCARDIAL INFARCTION}

Perioperative cardiovascular assessment of patients before noncardiac surgery may involve recommendations for postoperative surveillance to detect myocardial infarction. Numerous studies have examined postoperative cardiac biomarkers, but much of this literature no longer applies to current clinical practice with contemporary troponin assays. A widely accepted definition of acute myocardial infarction requires ischemic symptoms or ECG changes, in addition to elevations in troponin levels, to establish this diagnosis. Not uncommonly, troponin elevation alone is mistakenly equated with the diagnosis of myocardial infarction. Many conditions may be associated with elevated troponin levels, including sepsis, hypotension, renal insufficiency, pulmonary embolism, heart failure, and any other condition with increased ventricular wall stress.

In the postoperative setting, elevated troponin levels are far more likely to be caused by these other conditions than by acute myocardial infarction if ischemic symptoms or ECG changes are absent. For that reason, routine postoperative troponin measurements are not recommended in the ACC/AHA 2007 guidelines $^{3}$ for asymptomatic, stable patients who have undergone low-risk surgery. Furthermore, the current guidelines indicate that routine use of postoperative troponin measurements is not well established (ie, class IIb recommendation) even in patients undergoing intermediate-risk to high-risk noncardiac surgery. In patients who are symptomatic postoperatively or of considerable clinical concern, ECGs performed preoperatively, immediately postoperatively, and then for 2 consecutive days are probably the most effective approach to identifying myocardial ischemia. In patients with suspected cardiac symptoms or ECG changes suggestive of ischemia or infarction, serial measurements of troponin levels are recommended.

\section{CONCLUSION}

The ACC/AHA guidelines on perioperative cardiovascular evaluation of and care for patients undergoing noncardiac surgery have reflected the evolving literature since their initial publication in 1996. The 2007 revision of these guidelines gives greater emphasis than previous versions to preoperative clinical risk stratification, while deemphasizing routine preoperative cardiac testing in patients with known or suspected coronary heart disease. Judicious use of preoperative laboratory testing is warranted, but only if the results could substantially affect patient management.

$\beta$-Blocker therapy is a reasonable treatment choice for reducing nonfatal myocardial infarction and cardiac death during noncardiac surgery in higher-risk patients. Implementing such therapy, with appropriate dosage titration combined with vigilant perioperative administration and monitoring, remains a challenge for quality improvement in clinical practice.

Coronary revascularization remains an unproven management strategy for cardiac risk reduction during noncardiac surgery. In addition, coronary revascularization often introduces a number of management dilemmas and risks centered around interruption of antiplatelet therapy, particularly involving the use of thienopyridines after PCI with stenting. Further appropriately powered clinical trials are needed to definitively address the appropriate role and methodology of preoperative revascularization in patients with high-risk coronary heart disease.

\section{REFERENCES}

1. Eagle KA, Brundage BH, Chaitman BR, et al. Guidelines for perioperative cardiovascular evaluation for noncardiac surgery: report of the American College of Cardiology/American Heart Association Task Force on Practice Guidelines (Committee on Perioperative Cardiovascular Evaluation for Noncardiac Surgery). J Am Coll Cardiol. 1996;27(4):910-948.

2. Eagle KA, Berger PB, Calkins $\mathrm{H}$, et al. ACC/AHA guideline update for perioperative cardiovascular evaluation for noncardiac surgery-executive summary: a report of the American College of Cardiology/American Heart Association Task Force on Practice Guidelines (Committee to Update the 1996 Guidelines on Perioperative Cardiovascular Evaluation for Noncardiac Surgery) [published correction appears in J Am Coll Cardiol. 2006;47(11):2356]. J Am Coll Cardiol. 2002;39(3):542-553.

3. Fleisher LA, Beckman JA, Brown KA, et al. ACC/AHA 2007 guidelines on perioperative cardiovascular evaluation and care for noncardiac surgery: executive summary: a report of the American College of Cardiology/American Heart Association Task Force on Practice Guidelines (Writing Committee to Revise the 2002 Guidelines on Perioperative Cardiovascular Evaluation for Noncardiac Surgery). J Am Coll Cardiol. 2007;50(17):1707-1732.

4. Fleisher LA, Beckman JA, Brown KA, et al. ACC/AHA 2007 guidelines on perioperative cardiovascular evaluation and care for noncardiac surgery: a report of the American College of Cardiology/American Heart Association Task Force on Practice Guidelines (Writing Committee to Revise the 2002 Guidelines on Perioperative Cardiovascular Evaluation for Noncardiac Surgery). J Am Coll Cardiol. 2007;50(17):e159-e242. http://content.onlinejacc .org/cgi/content/full/j.jacc.2007.09.003. Accessed December 2, 2008.

5. Fleisher LA, Beckman JA, Brown KA, et al. ACC/AHA 2007 guidelines on perioperative cardiovascular evaluation and care for noncardiac surgery: a report of the American College of Cardiology/American Heart Association Task Force on Practice Guidelines (Writing Committee to Revise the 2002 Guidelines on Perioperative Cardiovascular Evaluation for Noncardiac Surgery). Circulation. 2007;116:e418-e500. http://circ.ahajournals.org/cgi /reprint/CIRCULATIONAHA.107.185699. Accessed December 2, 2008.

6. Gibbons RJ, Miller TD, Hodge D, et al. Application of appropriateness criteria to stress single-photon emission computed tomography sestamibi stud- 
ies and stress echocardiograms in an academic medical center. J Am Coll Cardiol. 2008;51(13):1283-1289.

7. Mehta R, Ward RP, Chandra S, Agarwal R, Williams KA. Evaluation of the American College of Cardiology Foundation/American Society of Nuclear Cardiology appropriateness criteria for SPECT myocardial perfusion imaging. J Nucl Cardiol. 2008 May-Jun;15(3):337-344. Epub 2008 Apr 16.

8. Douglas $\mathrm{P}$, Iskandrian AE, Krumholz HM, et al. Achieving quality in cardiovascular imaging: proceedings from the American College of CardiologyDuke University Medical Center Think Tank on Quality in Cardiovascular Imaging. J Am Coll Cardiol. 2006 Nov 21;48(10):2141-2151. Epub 2006 Nov 1.

9. Reilly DF, McNeely MJ, Doerner D, et al. Self-reported exercise tolerance and the risk of serious perioperative complications. Arch Intern Med. 1999;159(18):2185-2192.

10. Lee TH, Marcantonio ER, Mangione CM, et al. Derivation and prospective validation of a simple index for prediction of cardiac risk of major noncardiac surgery. Circulation. 1999;100(10):1043-1049.

11. Poldermans D, Bax JJ, Schouten O, et al; Dutch Echocardiographic Risk Evaluation Applying Stress Echo Study Group. Should major vascular surgery be delayed because of preoperative cardiac testing in intermediate-risk patients receiving beta-blocker therapy with tight heart rate control? J Am Coll Cardiol. 2006 Sep 5;48(5):964-969. Epub 2006 Aug 17.

12. Auerbach $\mathrm{AD}$, Goldman L. $\beta$-Blockers and reduction of cardiac events in noncardiac surgery: scientific review. JAMA. 2002;287(11):1435-1444.

13. Stevens RD, Burri $H$, Tramèr MR. Pharmacologic myocardial protection in patients undergoing noncardiac surgery: a quantitative systematic review. Anesth Analg. 2003;97(3):623-633.

14. Devereaux PJ, Beattie WS, Choi PT-L, et al. How strong is the evidence for the use of perioperative $\beta$ blockers in non-cardiac surgery? systematic review and meta-analysis of randomised controlled trials. BMJ. 2005 Aug 6;331(7512): 313-321. Epub 2005 Jul 4.

15. Hindler K, Shaw AD, Samuels J, Fulton S. Collard CD, Riedel B. Improved postoperative outcomes associated with preoperative statin therapy. Anesthesiology. 2006;105(6):1260-1272.

16. Poldermans D, Bax JJ, Kertai MD, et al. Statins are associated with a reduced incidence of perioperative mortality in patients undergoing major noncardiac vascular surgery. Circulation. 2003 Apr 15;107(14):1848-1851. Epub 2003 Apr 14

17. Durazzo AE, Machado FS, Ikeoka DT, et al. Reduction in cardiovascular events after vascular surgery with atorvastatin: a randomized trial. J Vasc Surg. 2004;39(5):967-975.

18. POISE Study Group. Effects of extended-release metoprolol succinate in patients undergoing non-cardiac surgery (POISE trial): a randomised controlled trial. Lancet. 2008 May 31;371(9627):1839-1847. Epub 2008 May 12.

19. Fleisher LA, Poldermans D. Perioperative $\beta$ blockade: where do we go from here? Lancet. 2008 May 31;371(9627):1813-1814. Epub 2008 May 12 .

20. McFalls EO, Ward HB, Moritz TE, et al. Coronary-artery revascularization before elective major vascular surgery. $N$ Engl J Med. 2004;351(27): 2795-2804.

21. Poldermans D, Schouten O, Vidakovic R, et al; DECREASE Study Group. A clinical randomized trial to evaluate the safety of a noninvasive approach in high-risk patients undergoing major vascular surgery: the DECREASE-V Pilot Study. J Am Coll Cardiol. 2007 May 1;49(17):1763-1769. Epub 2007 Apr 16.

22. Anderson JL, Adams CD, Antman EM, et al. ACC/AHA 2007 guidelines for the management of patients with unstable angina/non-ST elevation myocardial infarction: a report of the American College of Cardiology/American Heart Association Task Force on Practice Guidelines (Writing Committee to Revise the 2002 Guidelines for the Management of Patients With Unstable Angina/Non-ST-Elevation Myocardial Infarction) [published correction appears in J Am Coll Cardiol. 2008;51(9):974]. J Am Coll Cardiol. 2007;50(7): e1-e157.

23. Grines CL, Bonow RO, Casey DE Jr, et al. Prevention of premature discontinuation of dual antiplatelet therapy in patients with coronary artery stents: a science advisory from the American Heart Association, American College of Cardiology, Society for Cardiovascular Angiography and Interventions, American College of Surgeons, and American Dental Association, with representation from the American College of Physicians. J Am Coll Cardiol. 2007;49(6):734-739.

\section{The Symposium on Cardiovascular Diseases will continue in the February issue.}

